\title{
The Transitional Dynamics of an Endogenous Growth Model: Generalizing Production Functions
}

\author{
Manuel A. Gómez* \\ Universidad de A Coruña
}

\author{
Tiago Neves Sequeira ${ }^{\dagger}$ \\ Universidade da Beira Interior \\ INOVA, Universidade Nova de Lisboa
}

\begin{abstract}
This paper devises a class of endogenous growth models with physical capital, human capital and product varieties. Unlike previous literature, we introduce a general specification of production that allows for disentangling the gains from specialization from the monopolistic markup. The consequences of separating both parameters on the equilibrium dynamics of the model are analyzed. We find that the long-run growth rate ceases to depend on the markup when it is disentangled from the returns to specialization, and the same happens with the conditions for saddle-path stability. Numerical simulations show that the steady-state equilibria are more prone to be stable in the model that disentangles parameters. Furthermore, the model is able to generate a rich variety of development scenarios, accounting for a number of stylized facts related to the development process of industrialized countries.
\end{abstract}

JEL Classification: O31, O33, O41.

Key-Words: Endogenous growth; R\&D; Human capital; Stability; Markup.

\footnotetext{
*Departamento de Economía Aplicada II, Universidad de A Coruña, Campus de Elviña, 15071 A Coruña, Spain. email: mago@udc.es. I gratefully acknowledge financial support from the Spanish Ministry of Science and Innovation through Grant ECO2011-25490.

${ }^{\dagger}$ Departamento de Gestão e Economia. Universidade da Beira Interior. Estrada do Sineiro. 6200-209 Covilhã. email: sequeira@ubi.pt. I gratefully acknowledge financial support from PTDC/EGE-ECO 102238/2008 - FCT (Fundação para a Ciência e Tecnologia).
} 


\section{Introduction}

There is a broad consensus in the literature that physical capital accumulation, knowledge formation, and R\&D-based technological progress are the three main sources of growth. Arnold (2000a) and Funke and Strulik (2000) (AFS henceforth) have combined them into an endogenous growth model whose equilibrium dynamics has been analyzed by Gómez (2005). In the AFS model invention of new ideas depends solely and linearly on the share of human capital devoted to this activity. Recently, Sequeira (2011) and Gómez (2011a,b) (SG henceforth) have incorporated R\&D spillovers in innovation and an externality associated to the duplication of research effort, and have analyzed the equilibrium dynamics of the extended model.

The underlying structure of the AFS-SG model, in the tradition of Romer (1990), is such that the gains from specialization are rigidly linked to the elasticity of substitution between varieties, and thus, to the monopolistic markup in the economy. However, as argued e.g. by Kim (2004), there is no a-priori reason for such a restrictive link to exist. Following early work by Ethier (1982), a large literature has shown that breaking the connection between the gains of specialization and the markup has significant consequences on several issues as the persistence of technology shocks (Bénassy, 1996a), the optimal capital income tax (Coto-Martínez et al., 2007) and, specially, the optimality of R\&D investments (Bénassy, 1996b, 1998, de Groot and Nahuis, 1998, 2002, Jones and Williams, 2000, Alvarez-Pelaez and Groth, 2005, Sequeira and Reis, 2007). Separating these parameters also makes possible a more sensible calibration (e.g., Jones and Williams, 2000). However, aside from Sequeira and Reis (2007), none of these models include human capital accumulation as an engine of growth. Furthermore, most of them — and, in particular, Sequeira and Reis (2007) focus on analyzing the results in the steady state, so they do not solve for the transition dynamics.

This paper devises a model with physical capital accumulation, knowledge formation and increasing varieties in the line of the AFS-SG model. The fundamental difference is that, following Alvarez-Pelaez and Groth (2005), we introduce a general specification of production that allows for disentangling the gains from specialization from the elasticity of substitution between varieties and thus, from the markup. The R\&D technology is specified á la Jones (1995a) to allow for both R\&D spillovers in innovation and externalities associated to duplication of research. Hence, as particular cases, we consider the models with no externalities in R\&D (as in Arnold, 2000a, and Funke and Strulik, 2000), with only R\&D spillovers (as in Sequeira, 2011), with only duplication externalities (as in Gómez, 2011b, which also includes externalities in human capital accumulation) and with both types of externalities (as in Gómez, 2011a).

Our main objective is to analyze the equilibrium dynamics of the generalized model and, in particular, the effect of separating the gains from specialization from the markup. First, we provide conditions for the existence of a unique feasible steady-state equilibrium with positive long-run growth. Differently to what happened in the AFS-SG model — and also to the findings of Jones and Williams (2000) and Alvarez-Pelaez and Groth (2005) - , once the monopolistic markup is separated from the returns to specialization the long-run growth rate ceases to depend on the markup. We then study the (local) stability of the model. The transition dynamics is represented by a twodimensional stable manifold and, in spite of the complexity of the dynamic system, we provide a sufficient condition for stability - though the instability outcome cannot be ruled out. We find that the markup does not enter in the stability conditions of the general model, whereas it played a prominent role in the corresponding conditions for the SG model. A detailed numerical analysis shows that the introduction of spillovers in $R \& D$ causes a deep drop in the instability outcomes for the relevant set of parameters. The further introduction of the duplication externality decreases even more the instability cases and, in fact, we find that instability is quite rare in the general version of the model with both $R \& D$ spillovers and duplication externalities. Thus the increasing 
generalization of the $\mathrm{R} \& \mathrm{D}$ production structure brings about a noticeable increase in the stability outcomes of the steady state of the model. We also find that the steady-state equilibria of the model that disentangles parameters are more prone to be stable than those of the AFS-SG model.

Furthermore, we present some simulation results in order to gain some insight on the implied dynamics of the model. Using a typical calibration for the parameters set, the steady state is unstable when there are no external effects associated to the R\&D law of motion. When spillovers in $\mathrm{R} \& \mathrm{D}$ are introduced into the model, the steady state becomes saddle-path stable. Then, we show that the model is able to replicate the typical sequence of development in which education precedes innovation (e.g., Funke and Strulik, 2000) and also a transition in which innovation precedes knowledge formation. As recently argued by Iacopetta (2010), an innovation-education sequence could agree better than an education-innovation transition with the empirical fact that the rise in formal education to the masses follows rather than precedes the process of industrialization. Adding duplication externalities as well as R\&D spillovers, we show that the model is able to generate a realistic transition yielding a roughly constant growth rate during the last century, together with increasing time allocated to education and (more modestly than to education) to research. Furthermore, the model can also replicate a decreasing trend in the ratio between outputs and inputs to $R \& D$ while the value of patents increases more than wages.

This paper also contributes to a strand of the literature that investigates the transitional dynamics of endogenous growth models with R\&D. Arnold (2000b,c) proves that the steady states of both the socially-planned and the decentralized economies in the Romer (1990) model are saddle points. Eicher and Turnovsky $(1999,2001)$ develop a two-sector non-scale growth model and study the transition dynamics of the socially planned economy, whereas Steger (2005) studies its market equilibrium counterpart. Nevertheless, due to the complexity of the involved dynamic systems, a complete stability analysis is not provided. Arnold (2006) studies the dynamics of the Jones (1995a) model and proves that the economy converges in a saddle-path stable manner to the steady state along a two-dimensional stable manifold. Arnold and Kornprobst (2008) analyze the transition dynamics of the Romer (1990) model with quality upgrading à la Grossman and Helpman (1991) instead of increasing product variety. However, differently to ours, none of these models include human capital as a production factor, either in final goods production or in R\&D.

The rest of this paper is organized as follows. Section 2 describes the model. Section 3 analyzes the balanced growth equilibrium and its stability. Section 4 presents some numerical results. Section 5 concludes.

\section{The model}

We consider a closed economy inhabited by a constant population of identical representative agents. For simplicity, population is normalized to one, so that we may read all variables as per capita values.

\subsection{Production}

The final good, $Y$, which we take as numeraire, is produced with a Cobb-Douglas technology:

$$
Y=D^{\beta} H_{Y}^{1-\beta} n^{\eta}, \quad 0<\beta<1, \eta>0,
$$

where $H_{Y}$ is human capital allocated to the final good production, $D$ is an index of intermediate capital goods, and $n$ denotes the number of available varieties. Thus, the parameter $\eta$ captures the gains from specialization in the final good production. Following Alvarez-Pelaez and Groth (2005), 
the composite index $D$ is a CES aggregate of quantities of specialized capital goods:

$$
D=n\left(\frac{1}{n} \int_{0}^{n} x_{i}^{\alpha} d i\right)^{1 / \alpha},
$$

where $x_{i}$ is the intermediate capital good $i$, and $\alpha$ determines the elasticity of substitution between varieties. Intermediate goods are produced in a differentiated goods sector with physical capital, $K_{x}$ :

$$
x_{i}=K_{x_{i}} .
$$

Production of a new intermediate good requires the invention of a new blueprint. The production of new ideas is determined by human capital employed in R\&D labs and by the stock of disembodied knowledge $n$ according to the Jones (1995a) technology:

$$
\dot{n}=\bar{\epsilon} H_{n}=\epsilon \bar{H}_{n}^{\lambda-1} n^{\phi} H_{n}, \quad \epsilon>0,0<\lambda \leq 1,0 \leq \phi<1,
$$

where $H_{n}$ represents the allocation of human capital to $\mathrm{R} \& \mathrm{D}$ sector. The parameter $\phi$ measures the (positive) spillovers in R\&D. The term $\bar{H}_{n}$ represents average human capital devoted to innovation, so this specification incorporates the potential of (negative) externalities associated to the duplication of research effort.

The final good, $Y$, is used for consumption, $C$, and investment. For simplicity, we neglect depreciation of physical capital, which leads to the economy's resource constraint,

$$
Y=C+\dot{K}
$$

Human capital is accumulated according to

$$
\dot{H}=\xi H_{H}, \quad \xi>0,
$$

where $H_{H}$ represents the allocation of human capital to knowledge accumulation.

\subsection{Agents}

Human capital $H$ is supplied inelastically. Therefore, full employment requires that

$$
H=H_{H}+H_{n}+H_{Y}=\left(u_{H}+u_{n}+u_{Y}\right) H
$$

where $u_{H}, u_{n}$ and $u_{Y}$ are the shares of human capital allocated to human capital accumulation, $\mathrm{R} \& \mathrm{D}$ activities and the final good production, respectively.

The representative agent earns wages, $w$, per unit of employed human capital, $H-H_{H}$. He also earns returns, $r$, per unit of aggregate wealth, $W$, which leads to a budget constraint $\dot{W}=$ $r W+w\left(H-H_{H}\right)-C$. Subject to this constraint and the knowledge formation technology (6), he maximizes the intertemporal utility

$$
U=\int_{0}^{\infty} \frac{C^{1-\theta}-1}{1-\theta} e^{-\rho t} d t
$$

where $\rho>0$ denotes the time preference rate, and $1 / \theta>0$ is the elasticity of intertemporal substitution. Agents take the aggregate rate of innovation as given. 

yield $^{1}$

Let $g_{x}$ denote $x$ 's growth rate, $g_{x}=\dot{x} / x$. The first order conditions for an interior solution

$$
\begin{aligned}
& g_{C}=(r-\rho) / \theta, \\
& g_{w}=r-\xi .
\end{aligned}
$$

The first of these equations is the standard Ramsey rule. The second indicates that the growth rate of wages must be sufficiently high compared to the interest rate in order to ensure investment in human capital.

\section{$2.3 \quad$ Firms and markets}

The markets for the final good and its inputs are perfectly competitive. This implies that

$$
\begin{aligned}
w & =(1-\beta) Y / H_{Y}, \\
p_{D} & =\beta Y / D,
\end{aligned}
$$

where $p_{D}$ represents the price for the index of intermediate capital goods.

The demand for each intermediate good results from the maximization of profits in the final good sector. Each firm in the intermediate goods sector owns an infinitely-lived patent for selling its variety $x_{i}$. Producers act under monopolistic competition and maximize operating profits,

$$
\pi_{i}=\left(p_{i}-r\right) x_{i}
$$

where $p_{i}$ denotes the price of intermediate good $i$ and $r$ is the unit cost of $x_{i}$. Profit maximization in this sector implies that each firm charges a price of

$$
p_{i}=p=r / \alpha \text {. }
$$

With identical technologies and symmetric demand, the quantity supplied is the same for all goods, $x_{i}=x$. Hence, Eq. (2) can be written as

$$
D=n x=K,
$$

using the fact that intermediate goods are produced with capital goods. Therefore, combining (1) and (15), the production function can be re-written as

$$
Y=K^{\beta} H_{Y}^{1-\beta} n^{\eta}
$$

Hence, differently to the AFS-SG model, this specification allows for disentangling the gains from specialization, $\eta$, from the markup, $1 / \alpha .^{2}$

From $p_{D} D=p x n$, together with equations (12) and (14), we obtain that

$$
x n=K=\alpha \beta Y / r .
$$

After insertion of (14) and (17) into (13), profits are given by

$$
\pi=(1-\alpha) \beta Y / n .
$$

\footnotetext{
${ }^{1}$ We shall focus on a fully industrialized economy with both education and innovation.

${ }^{2}$ In the AFS-SG model, production of the final good in equilibrium is given by $Y^{1-\eta}=(\alpha \eta)^{\eta} K^{\beta} H_{Y}^{1-\beta-\eta} n^{(1-\alpha) \eta / \alpha}$.
} 
An innovation is worth the present value of the stream of monopoly profits, $v(t)=\int_{t}^{\infty} e^{-R(\tau, t)} \pi(\tau) d \tau$, with $R(\tau, t)=\int_{t}^{\tau} r(s) d s$. Differentiating this expression with respect to time yields the no-arbitrage equation

$$
g_{v}=r-\pi / v
$$

Finally, in an equilibrium with innovation, free-entry into R\&D requires ${ }^{3}$

$$
w H_{n}=v \bar{\epsilon} H_{n}=v \epsilon \bar{H}_{n}^{\lambda-1} n^{\phi} H_{n} .
$$

\subsection{Equilibrium dynamics}

Henceforth we shall take into account that $\bar{H}_{n}=H_{n}$ in equilibrium. As usual, we shall express the dynamics of the economy in terms of variables that are constant in the steady state. ${ }^{4}$ Let $\chi \equiv C / K$ denote the consumption to physical capital ratio, and $\psi \equiv H^{\lambda} / n^{1-\phi}$, the knowledge-ideas ratio. Using (17), the economy's resource constraint (5) can be rewritten as

$$
g_{K}=\frac{1}{\alpha \beta} r-\chi .
$$

Some equations will be needed for solving the model. Log-differentiating (11) and (16) and solving for $g_{u_{Y}}$, we obtain

$$
g_{u_{Y}}=-\frac{1}{\beta} g_{w}+\frac{\eta}{\beta} g_{n}+g_{K}-g_{H}
$$

Log-differentiating (4) yields

$$
g_{g_{n}}=\lambda\left(g_{u_{n}}+g_{H}\right)-(1-\phi) g_{n} .
$$

Furthermore, log-differentiating (20) and substituting $g_{w}$ from (10), $g_{v}$ from (19), $\pi$ from (18), $v$ from (20) and $w$ from (11) we get

$$
(\lambda-1)\left(g_{u_{n}}+g_{H}\right)=-\xi+\frac{(1-\alpha) \beta u_{Y}}{(1-\beta) u_{n}} g_{n}-\phi g_{n} .
$$

If $\lambda<1$ and $0 \leq \phi<1$, the dynamics of the economy in terms of the variables $r, \chi, u_{P}, \psi$ and $g_{n}$ is determined by the following fifth-order system:

$$
\begin{aligned}
g_{\chi} & =\left(\frac{1}{\theta}-\frac{1}{\alpha \beta}\right) r+\chi-\frac{\rho}{\theta}, \\
g_{r} & =-\frac{1-\beta}{\beta}(r-\xi)+\frac{\eta}{\beta} g_{n}, \\
g_{u_{Y}} & =\left(\frac{1-\alpha}{\alpha \beta}\right) r+\frac{\eta}{\beta} g_{n}-\chi-\xi\left(1-u_{Y}-\epsilon^{-1 / \lambda} \psi^{-1 / \lambda} g_{n}^{1 / \lambda}\right)+\frac{\xi}{\beta}, \\
g_{\psi} & =\lambda \xi\left(1-u_{Y}-\epsilon^{-1 / \lambda} \psi^{-1 / \lambda} g_{n}^{1 / \lambda}\right)-(1-\phi) g_{n},
\end{aligned}
$$

\footnotetext{
${ }^{3}$ Alternatively, proceeding in a similar way to Iacopetta (2011), we could consider that the agent is subject to the dynamic budget constraint $\dot{W}=r W+w u_{Y} H+v \dot{n}-C$, where $v \dot{n}$ accounts for the entrepreneurial gain of establishing new intermediate good firms. The agent would then seek to maximize the utility function (8) subject to the budget constraint, the constraint on human capital accumulation (7), and the flow of innovation (4). From the first-order conditions of this problem, we would also get (9), (10) and (20).

${ }^{4}$ Making a change of variables to analyze the dynamic properties of balanced growth paths is a standard practice in the endogenous growth literature (e.g., Benhabib and Perli, 1994, Barro and Sala-i-Martin, 1995, Benhabib et al., 2000, Mino, 2001, and Arnold, 2000b, 2006).
} 


$$
g_{g_{n}}=-\frac{\lambda(1-\alpha) \beta}{(1-\beta)(1-\lambda)} u_{Y} \epsilon^{1 / \lambda} \psi^{1 / \lambda} g_{n}^{1-1 / \lambda}+\frac{\lambda \xi}{1-\lambda}-g_{n}+\frac{\phi}{1-\lambda} g_{n}
$$

We have used that $u_{H}=1-u_{Y}-u_{n}$ and that $u_{n}=\epsilon^{-1 / \lambda} \psi^{-1 / \lambda} g_{n}^{1 / \lambda}$ from (4). Combining (9) and (21) we obtain (25). Using that $g_{r}=g_{Y}-g_{K}$ from (17) and combining the growth rate version of (11) and (16) with (10), we get (26). Eq. (27) results from (22), using (10), (21) and (6). Eq. (28) is obtained by using the definition of $\psi$. Substituting (24) in (23), we get (29).

If $\lambda=1$ and $0<\phi<1$, from (24) and using (4), which becomes $g_{n}=\epsilon u_{n} \psi$, we get

$$
g_{n}=\frac{1}{\phi}\left[\frac{(1-\alpha) \beta \epsilon u_{Y} \psi}{1-\beta}-\xi\right] .
$$

Thus, the dynamics of the economy is driven by the system (25)-(28), where $g_{n}$ should be substituted with (30).

Finally, if $\lambda=1$ and $\phi=0$, from (24) and using that $g_{n}=\epsilon u_{n} \psi$ from (4), we get

$$
u_{Y}=\frac{(1-\beta) \xi}{(1-\alpha) \beta \epsilon \psi} .
$$

Hence, $g_{u_{Y}}=g_{n}-g_{H}$, which combined with (27) entails that

$$
g_{n}=\frac{(1-\alpha) r-\alpha \beta \chi+\alpha \xi}{\alpha(\beta-\eta)} .
$$

Thus, if $\lambda=1$ and $\phi=0$, the evolution of the economy is described by the system (25), (26) and (28), where $u_{Y}$ and $g_{n}$ should be replaced with (31) and (32), respectively.

\section{Steady state: existence and stability}

In this section, we focus on a balanced growth path (or steady state) equilibrium in which all variables grow at constant but possibly different rates, and the shares of human capital in its different uses are constant.

\subsection{Existence}

The following proposition provides conditions for the existence of a unique feasible steady state with positive long-run growth. ${ }^{5}$

Proposition 1. Let $\xi>\rho$. The economy has a unique positive steady-state equilibrium with positive long-run growth, in which the interest rate is

$$
\hat{r}=\frac{(1+M) \theta \xi-\rho}{(1+M) \theta-1}
$$

the ratio of consumption to physical capital is

$$
\hat{\chi}=\left(\frac{1}{\alpha \beta}-\frac{1}{\theta}\right) \hat{r}+\frac{\rho}{\theta}
$$

\footnotetext{
${ }^{5}$ The fact that this proposition is true for $0<\lambda \leq 1$ and $0 \leq \phi<1$ results evident from its proof.
} 
the long-run growth rate of intermediates is

$$
\hat{g}_{n}=\frac{\lambda M(\xi-\rho)}{(1-\phi)[(1+M) \theta-1]},
$$

the long-run growth rate of human capital is

$$
\hat{g}_{H}=(1-\phi) \hat{g}_{n} / \lambda,
$$

the share of human capital devoted to RED and production can be obtained from

$$
\begin{gathered}
\hat{u}_{n}=\frac{(1-\alpha) \beta \hat{g}_{n}}{(1-\beta)\left(\xi-\hat{g}_{H}+\hat{g}_{n}\right)+(1-\alpha) \beta \hat{g}_{n}}\left(1-\frac{\hat{g}_{H}}{\xi}\right), \\
\hat{u}_{Y}=1-\hat{u}_{n}-\frac{\hat{g}_{H}}{\xi}
\end{gathered}
$$

the knowledge-ideas ratio is given by

$$
\hat{\psi}=\frac{\hat{g}_{n}}{\epsilon \hat{u}_{n}^{\lambda}},
$$

and the long-run growth rate of income, consumption, and physical capital is

$$
\hat{g}_{Y}=\hat{g}_{C}=\hat{g}_{K}=(1+1 / M) \hat{g}_{H},
$$

where $M=(1-\beta)(1-\phi) /(\eta \lambda)$, if and only if

$$
\theta>\theta_{\min }=\frac{1+M(1-\rho / \xi)}{1+M} .
$$

Proof. In the Appendix.

It should be noted that a simple sufficient condition for the condition (41) to hold is $\theta \geq 1$. The former Proposition shows that long-run growth does not depend on the market power parameter $\alpha$, as in the Jones (1995a) model. This result is in sharp contrast with the AFS-SG model (see, e.g., Gómez, 2011a, Proposition 1), in which it can be easily shown that the long-run growth rate of income decreases as $\alpha$ increases, whereas the long-run growth rates of human capital and intermediates are increasing, decreasing or constant as $\alpha$ increases depending on whether $\theta>1$, $\theta<1$ or $\theta=1$, respectively. Thus, the growth effect of the elasticity of substitution between varieties - and, therefore, of the markup - in the AFS-SG model results from its link to gains from specialization. Furthermore, our invariance result also differs from the findings in Jones and Williams (2000) and Alvarez-Pelaez and Groth (2005), where the long-run growth rate of income - in the market economy - decreases as the elasticity of substitution in intermediates $\alpha$ increases.

\subsection{Stability: analytical results}

Now, we study the equilibrium stability in the neighbourhood of the steady state. As usual, we assume that the stocks of physical capital, human capital and the number of varieties move sluggishly, so that $K(0), H(0)$ and $n(0)$ are given by their historical values. The analysis of the linearized system around the steady state will establish that the system that drives the dynamics of the economy features two state-like variables. Therefore, we will look for conditions that ensure the presence of two stable roots, so that the stable manifold is two-dimensional. Henceforth, we will say that the steady state is unstable when the dimension of the stable manifold - the number of 
stable roots of the linearized dynamics - is less than two - the number of predetermined variables. In this case, the system that describes the dynamics of the model has "too many" unstable roots, so it is not possible to make the system stable for arbitrary initial values of the predetermined variables. ${ }^{6}$

We shall first analyze the stability of the steady state in the general model with both $R \& D$ spillovers and duplication externalities, $\lambda<1$ and $0<\phi<1$, and in the model with only duplication externalities, $\lambda<1$ and $\phi=0$. The following proposition proves that the steady state is either saddle-path stable or unstable, and provides a simple sufficient condition to rule out instability.

Proposition 2. If $\lambda<1$ and $0 \leq \phi<1$, in the conditions of Proposition 1:

a) the steady-state equilibrium is either saddle-path stable or unstable,

b) a sufficient condition to rule out the instability outcome is

$$
\beta(1-\phi) \geq \eta \lambda .
$$

Proof. In the Appendix.

The following example shows that instability cannot be ruled out in the general model.

Example 1. The parameterization $\beta=0.80, \eta=1.64, \alpha=0.99, \xi=0.0274, \rho=0.023, \theta=1.25$, $\phi=0.95$ and $\lambda=0.95$ yields the (feasible) steady state: $\hat{r}=0.04425, \hat{\chi}=0.0389, \hat{u}_{Y}=0.9932$, $\hat{u}_{H}=0.0040, \hat{u}_{n}=0.0028, \hat{g}_{n}=0.0021, \hat{z}=0.5292, \hat{g}_{H}=0.0001$ and $\hat{g}_{Y}=\hat{g}_{K}=0.017$. The eigenvalues of the linearized system are $0.0455 \pm 0.0330 i, 0.0015 \pm 0.0162 i$ and 0.02725 and, therefore, the steady state is unstable.

In the case that there are only spillovers in $\mathrm{R} \& \mathrm{D}, \lambda=1$ and $0<\phi<1$, the following proposition studies the stability of the steady state.

Proposition 3. If $\lambda=1$ and $0<\phi<1$, in the conditions of Proposition 1:

a) the steady-state equilibrium is either saddle-path stable or unstable,

b) a sufficient condition to rule out the instability outcome is

$$
\beta(1-\phi) \geq \eta
$$

Proof. In the Appendix.

To complete the former result, the following example shows that the instability outcome cannot be ruled out. ${ }^{7}$

Example 2. The parameterization $\beta=0.80, \eta=1.64, \alpha=0.99, \xi=0.0316, \rho=0.023, \theta=1.5$, $\phi=0.95$ and $\lambda=1$ yields the (feasible) steady state: $\hat{r}=0.0485, \hat{\chi}=0.0442, \hat{u}_{Y}=0.9943$, $\hat{u}_{H}=0.0033, \hat{u}_{n}=0.0024, \hat{g}_{n}=0.0021, \hat{z}=0.8390, \hat{g}_{H}=0.0001$ and $\hat{g}_{Y}=\hat{g}_{K}=0.017$. The eigenvalues of the linearized system are $0.0061 \pm 0.0233 i, 0.0901$ and 0.0315 and, therefore, the steady state is unstable.

\footnotetext{
${ }^{6}$ Note that the change of variables made satisfy the conditions (7)-(9) stated in Martínez-García (2003), -i.e., the new variables explain their own dynamics, as it happens in the dynamic system (25)-(29), when this system is evaluated at the balanced growth path the result is a steady state, and they explain the growth rates of the state and costate variables - , as well as the conditions in her Lemmas 1 and 2. Hence, analyzing the existence of paths converging to the balanced growth path is equivalent to studying the stability of the steady state in (25)-(29).

${ }^{7}$ Note that, in this case, the dynamics of the economy are driven by the system (25)-(28), where $g_{n}$ should be substituted with (30).
} 
In the simplest case in which there are neither R\&D spillovers nor duplication externalities, $\lambda=1$ and $\phi=0$, we can state the following proposition.

Proposition 4. Let $\lambda=1$ and $\phi=0$. In the conditions of Proposition 1, the steady state is saddle-path stable if and only if $\eta>\beta$ and one of the following conditions is verified:

i) $\alpha(1-\beta+\eta) \leq 1$, or

ii) $\alpha(1-\beta+\eta)>1$ and $\theta<\theta_{0}$, where

$$
\theta_{0}=\frac{\alpha(1-\beta+\eta)[\xi(\beta-\eta)-\beta \rho]+\eta \rho}{\xi[1-\alpha(1-\beta+\eta)](1-\beta+\eta)} .
$$

Proof. In the Appendix.

Thus, in the simplest model with no externalities in $\mathrm{R} \& \mathrm{D}, \lambda=1$ and $\phi=0$, we have been able to establish a necessary and sufficient condition for saddle-path stability of the steady state. However, given the complexity of the dynamic system that leads the economy, we could devise only sufficient conditions for stability in the most general model with R\&D spillovers and duplication externalities, $\lambda<1$ and $0<\phi<1$, in the model with only duplication externalities, $\lambda<1$ and $\phi=0$, and in the model with only R\&D spillovers, $\lambda=1$ and $0<\phi<1$. Interestingly, we can observe that in these models the markup does not enter in the stability condition, whereas it played a prominent role in the corresponding conditions for the SG model. ${ }^{8}$ This fact suggests that the influence of the markup on the stability conditions in the SG model could be caused more by its link with gains from specialization than by its own relevance. Therefore, it would be interesting to compare the stability properties of the present model with those of the AFS-SG model.

\subsection{Stability: a quantitative assessment}

The former section has studied analytically the conditions for saddle-path stability of the steady state. However, the theoretical results obtained do not allow to derive neat conclusions on the effect that introducing external effects in $R \& D$ has on the stability of the steady state. Therefore, we will make a numerical experiment to compare the stability properties in the different models. Furthermore, we will compare the results with those attainable in the AFS-SG model to investigate the consequences of disentangling the gains from specialization from the markup. To this end, we construct a grid of plausible values for the parameters. For each of the cases considered we compute the eigenvalues of the linearization of the dynamic system that drives the economy. We then calculate the percentage of instable cases; i.e., the number of cases in which we obtain less than two stable roots, over the number of total cases considered.

Table 1 presents our assumptions about the parameter space considered. We keep fixed the preference parameters $\theta=2$ and $\rho=0.023$, which are standard values (e.g., Funke and Strulik, 2000, Gómez, 2005, Sequeira, 2011). ${ }^{9}$ The share of physical capital $\beta$ begins in 0.1 and ends at 0.7 , so that it covers the range of plausible values for this parameter. The market power parameter $\alpha$ varies between 0.2 and 0.95 which comprises with a wide margin the range of empirical estimates obtained by Norrbin (1993) and Basu (1996). The parameter that measures gains from specialization $\eta$ takes values in the interval 0.1-1.65, which is in the range considered by Jones and Williams (2000). We leave the parameters $\phi$ and $\lambda$ to take values in a wide interval between 0.1 and 0.95 in the general model. Of course, in the model without externalities, we set $\phi=0$ and $\lambda=1$, and in the model

\footnotetext{
${ }^{8}$ The sufficient stability condition in the SG model is $\alpha \beta(1-\phi) \geq(1-\alpha) \eta \lambda$ (see Gómez, 2011a, and Gómez and Sequeira, 2011).

${ }^{9}$ Extensive simulation has shown that our conclusions do not change if different plausible values of these parameters are considered.
} 
Table 1: The instability ratio in the new model

\begin{tabular}{|c|c|c|c|c|c|c|c|}
\hline Parameters & $\rho$ & $\theta$ & $\beta$ & $\eta$ & $\alpha$ & $\phi$ & $\lambda$ \\
\hline Range & 0.023 & 2 & {$[0.1,0.7]$} & {$[0.1,1.65]$} & {$[0.2,0.95]$} & {$[0.1,0.95]$} & {$[0.1,0.95]$} \\
\hline R\&D function & \multicolumn{3}{|c|}{$\phi=0, \lambda=1$} & \multicolumn{2}{|c|}{$0<\phi<1, \lambda=1$} & \multicolumn{2}{|c|}{$0<\phi<1, \lambda<1$} \\
\hline Step & \multicolumn{3}{|c|}{0.01} & \multicolumn{2}{|c|}{0.025} & \multicolumn{2}{|c|}{0.05} \\
\hline Instable cases & \multicolumn{3}{|c|}{323628} & \multicolumn{2}{|c|}{26933} & \multicolumn{2}{|c|}{0} \\
\hline Total & \multicolumn{3}{|c|}{718589} & \multicolumn{2}{|c|}{1708875} & \multicolumn{2}{|c|}{2156544} \\
\hline$\%$ Instable cases & \multicolumn{3}{|c|}{$45.04 \%$} & \multicolumn{2}{|c|}{$1.58 \%$} & & $0 \%$ \\
\hline
\end{tabular}

with only $\mathrm{R} \& \mathrm{D}$ spillovers, $\lambda=1$. As a sort of normalization, the parameter $\xi$-not reported in the Table - is set so that the long-run growth rate of income is equal to 1.7 percent in all the cases. ${ }^{10}$ Table 1 also reports the step taken between consecutive values of the parameters in the range considered. In the model with no externalities in $R \& D$ the step between consecutive values of the parameters is 0.01 ; in the model with only $\mathrm{R} \& \mathrm{D}$ spillovers is 0.025 , and in the model with duplication externalities as well the step is $0.05 .^{11}$ As Table 1 shows, the probability of saddlepath stability of the steady state increases significantly with the introduction of externalities in the R\&D sector. A big drop in instability cases is observed when the model incorporates R\&D spillovers - from $45.04 \%$ to $1.58 \%$-, with a smaller drop when the model incorporates duplication externalities as well - from $1.58 \%$ to zero. ${ }^{12}$

Table 2: The instability ratio in the AFS-SG model

\begin{tabular}{|c|c|c|c|c|c|c|c|}
\hline Parameters & $\rho$ & $\theta$ & $\beta$ & $\eta$ & $\alpha$ & $\phi$ & $\lambda$ \\
\hline Range & 0.023 & 2 & {$[0.1,0.7]$} & {$[0.1,0.95-\beta]$} & {$[0.2,0.95]$} & {$[0.1,0.95]$} & {$[0.1,0.95]$} \\
\hline R\&D function & \multicolumn{3}{|c|}{$\phi=0, \lambda=1$} & \multicolumn{2}{|c|}{$0<\phi<1, \lambda=1$} & \multicolumn{2}{|c|}{$0<\phi<1, \lambda<1$} \\
\hline Step & \multicolumn{3}{|c|}{0.01} & \multicolumn{2}{|c|}{0.025} & \multicolumn{2}{|c|}{0.05} \\
\hline Instable cases & \multicolumn{3}{|c|}{167122} & \multicolumn{2}{|c|}{37880} & \multicolumn{2}{|c|}{0} \\
\hline Total & \multicolumn{3}{|c|}{213076} & \multicolumn{2}{|c|}{515375} & \multicolumn{2}{|c|}{673920} \\
\hline$\%$ Instable cases & \multicolumn{3}{|c|}{$78.43 \%$} & \multicolumn{2}{|c|}{$7.35 \%$} & & $0 \%$ \\
\hline
\end{tabular}

In order to compare the stability properties of the present model with those of the AFS-SG model, Table 2 reports the results of a similar exercise to that described above. However, given that in the AFS-SG model the elasticity of human capital in the final good production is $1-\beta-\eta$, we consider $0.95-\beta$ as an upper bound of $\eta$, so that the elasticity of human capital is at least 0.05. As in the former case, the probability of stability increases significantly with the introduction of externalities associated to R\&D. However, comparing the results displayed in Tables 1 and 2,

\footnotetext{
${ }^{10}$ Given that $\xi>\rho$ and $\theta>1>\theta_{\min }$, Proposition 1 ensures that there exists a unique and positive steady state for all the parameters configurations considered.

${ }^{11}$ We consider higher steps as we extend the model to incorporate spillovers in R\&D and duplication externalities because of the huge increase in the number of cases to be considered.

${ }^{12}$ Of course, as shown in Example 1, this does not mean that instability can be ruled out but only that it is highly unlikely.
} 
we observe that for the two models without duplication externalities $(\lambda=1)$, the steady-state equilibria of the present model — which disentangle parameters - are more prone to be stable than those of the AFS-SG model. In the model without R\&D externalities, from an 'instability ratio' of almost $80 \%$ in the AFS-SG model, the ratio drops to $45 \%$ in the model with a more general functional form for the final good production. In the model with spillovers in R\&D the drop is still important, from $7.35 \%$ to $1.58 \%$. Concerning the comparison of the most general model —with both externalities in the R\&D sector-, it is not possible to reach a clear conclusion as the exercise yields no instability cases in both of them. This suggests that instability is highly unlikely in both models.

\section{Simulation results}

In order to gain insight on the transition dynamics generated by the model, this section presents some simulation results. The benchmark parameterization and the implied steady-state values are displayed in Table 3. Preference parameters, $\theta$ and $\rho$, are standard (e.g., Funke and Strulik, 2000, Gómez, 2005, Sequeira, 2011). Technology parameters in the final good production, $\beta$ and $\eta$, and the markup, $1 / \alpha$, are taken from Jones and Williams (2000). Regarding the R\&D technology parameters, we could consider as a benchmark the case in which there are no externalities associated to $\mathrm{R} \& \mathrm{D}, \lambda=1$ and $\phi=0$. However, for this calibration the steady state is unstable because the stability condition stated in Proposition 4 is not satisfied as $\beta>\eta$. Therefore, we first consider the case with R\&D spillovers and no duplication externalities, $\lambda=1$ and $\phi>0$, and follow Jones and Williams (2000) to set $\phi=0.457 .{ }^{13}$ Then we introduce mild duplication externalities into the model, $\lambda=0.9$, in order to illustrate its effect on the transition dynamics. In both cases the productivity parameter in human capital accumulation, $\xi$, is adjusted so that the long-run growth rate of income is equal to 1.7 percent. Table 3 shows that the steady-state shares of time devoted to work, education and innovation, as well as the interest rate, have plausible values.

Let us first consider the Case 1 in which there are R\&D spillovers and no duplication externalities, $\lambda=1$ and $\phi=0.457$. Figures 1 and 2 display two possible transition dynamics computed by backward integration (e.g., Brunner and Strulik, 2002, and Atolia and Buffie, 2009). Several features of the displayed dynamics merit attention. First, the model can generate an educationinnovation development sequence in which human capital accumulation precedes R\&D (Figure 1) or and innovation-education sequence in which innovation precedes knowledge accumulation (Figure 2). ${ }^{14}$ Funke and Strulik (2000) suggested that the typical phases of development are characterized in a temporal order by physical capital accumulation, knowledge formation and innovation. However, historical data examined by Galor (2005) shows that in the first phase of the Industrial Revolution, human capital played a limited role in the production process. In the second phase of the Industrial Revolution, the increasing pace of technological progress ultimately brought about an industrial demand for human capital that stimulated human capital formation. Therefore, a sequence in which innovation precedes education could agree with historical evidence as suggested by Iacopetta (2010). He also shows that the AFS model is able to generate transition dynamics in which innovation precedes education as well as the typical education-innovation sequence. ${ }^{15}$ Figures 1 and 2 show that our model is also able to generate both sequences of the phases of economic

\footnotetext{
${ }^{13}$ This relatively low value for $\phi$ is also adequate for a model with human capital accumulation, as argued by Sequeira and Reis (2007).

${ }^{14}$ We also depict the time path of the variable $z \equiv \epsilon^{1 / \lambda} \psi^{1 / \lambda} u_{Y}$, which is used to analyze the stability of the steady state (see Appendix).

${ }^{15}$ However, numerical results recently provided by Gómez (2011b) cast doubts on the ability of the AFS model to describe the development process in a realistic fashion.
} 
Table 3: Parameter and steady-state values

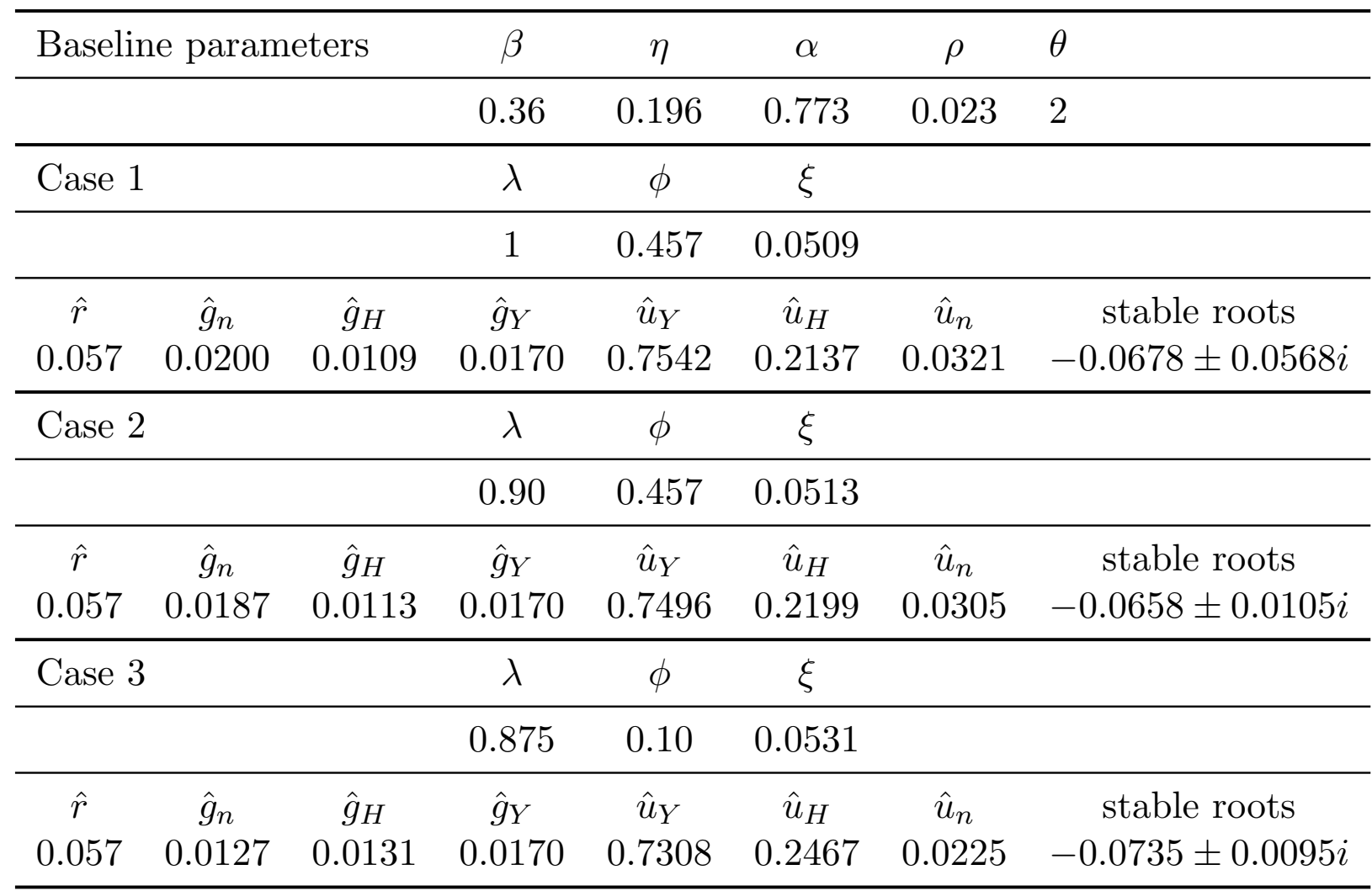

development. Thus, it is sufficiently general to encompass different possible trajectories of modern economies as, e.g., the British Industrial Revolution in which innovation preceded education of masses and other later industrialization experiences in which investments in education may have preceded large-scale innovation investments.

Now, let us introduce duplication externalities in the model as well. To this end, we set $\lambda=0.9$ and keep constant the remaining parameters (aside from $\xi$ ). The dynamics of the economy is depicted in Figure 3. The model generates transitional dynamics in which innovation precedes knowledge formation which, as discussed above, accords with the empirical fact that the rise in formal education to the masses follows rather than precedes the process of industrialization. ${ }^{16}$ As it can be observed, the implied transition paths exhibit a more realistic behaviour. First, the economy starts with a low time devoted to innovation. As the economy evolves, innovation time increases steadily, and expands hand in hand with educational time at early stages of development. This behaviour agrees with empirical evidence reported, e.g., by Jones (1995b, 2002). Second, the growth rate of income remains relatively constant (with a very smooth increasing trend) along the transition, which is also in accordance with data (e.g., Jones, 1995b, Stokey and Rebelo, 1995, Maddison, 2001).

Figure 3 also shows that, as the pace of increase in technologies is low when compared with the pace of the education rise, the facts according to which outputs of $R \& D$ increase less than inputs and according to which the value of patents is increasing more than wages (see e.g. Kortum, 1993) are verified during the initial transition of the economy. However, the verification of these

\footnotetext{
${ }^{16}$ Actually, the condition for no time devoted to innovation, $w H_{n}>v \epsilon H_{n}^{\lambda} n^{\phi}$, cannot be satisfied if $\lambda<1$, so that the model with duplication externalities cannot generate an education-innovation sequence.
} 

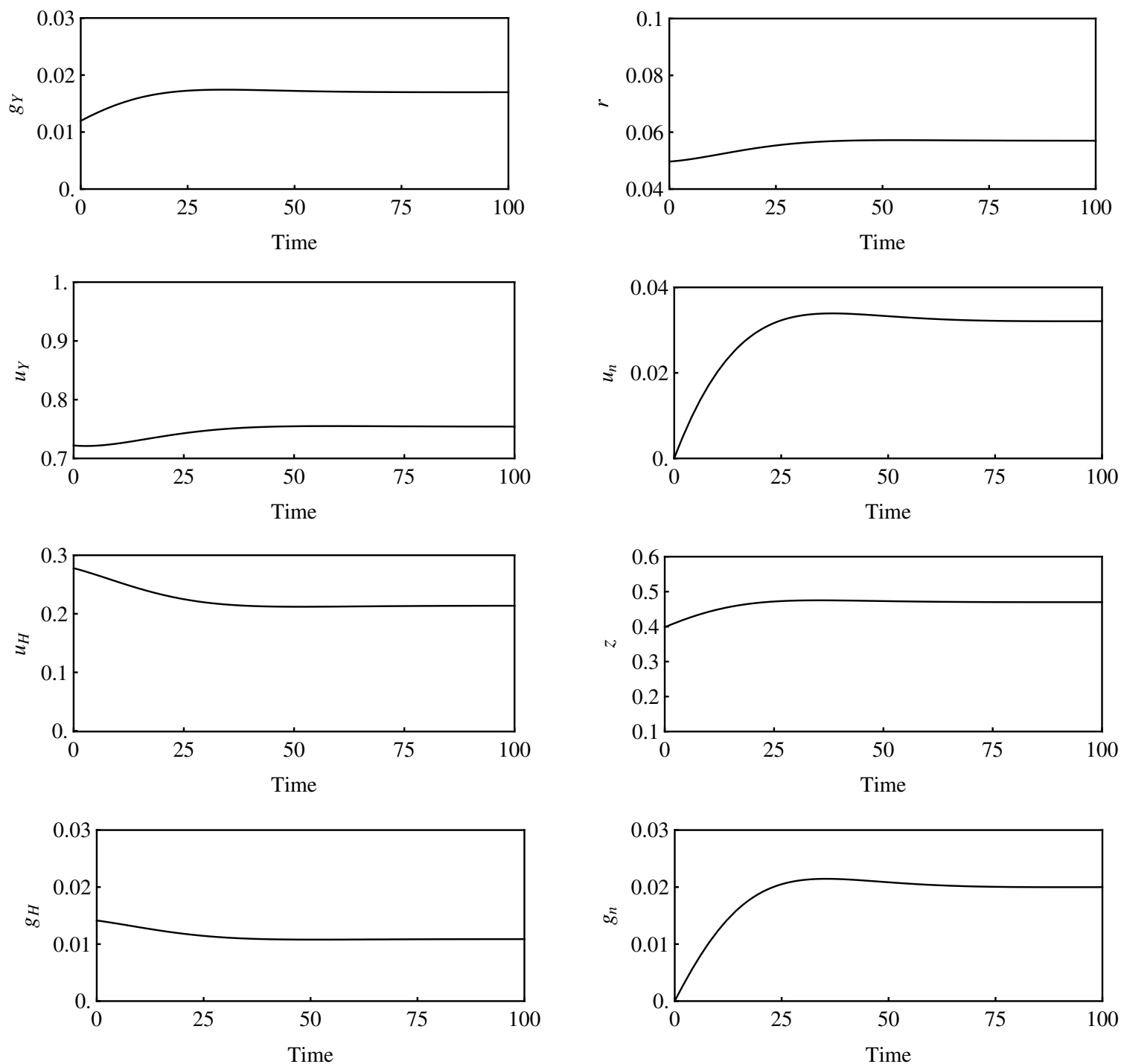

Figure 1: Time paths of representative variables in the model without duplication externalities: education-innovation sequence.

Note: Parameter values are shown in Case 1 of Table 3.

facts in the balanced growth path may require a slightly higher duplication and a lower spillover externalities. The set of parameter values shown in Case 3 allows for the verification of these facts. In Figure 4, the pace of variables is qualitatively similar to the pace shown in Figure 3. Even though the economy begins with a positive technology growth rate, the human capital growth rate rapidly overcomes it and ends out higher, which allows for the replication of the R\&D-related facts mentioned above. Intuitively, as the quantity of technologies grows less than human capital, the ratio between outputs and inputs to the $\mathrm{R} \& \mathrm{D}$ sector decreases (both considering physical units or expenditure to measure inputs). Meanwhile, the value of technologies is growing more than the value of human capital (wages). 

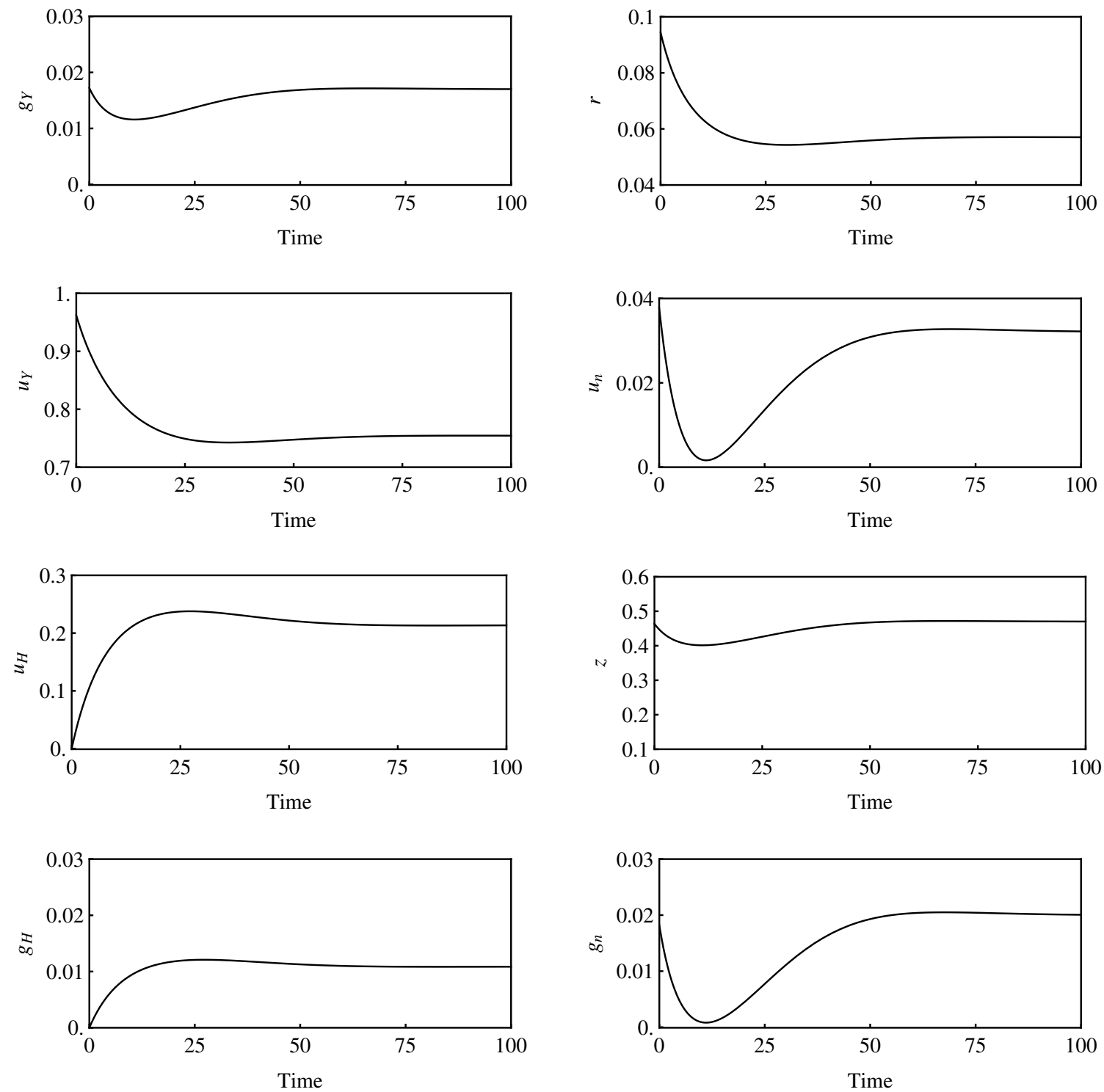

Figure 2: Time paths of representative variables in the model without duplication externalities: innovation-education sequence.

Note: Parameter values are shown in Case 1 of Table 3.

\section{Conclusion}

This paper devises a class of endogenous growth models with physical capital, human capital and R\&D. Innovation may be subject to the presence of spillovers in R\&D and/or externalities associated to the duplication of research effort. The main novelty with respect to the previous literature is that the production structure allows for disentangling the gains from specialization from the elasticity of substitution between varieties, and thus, from the markup. Separating both parameters is shown to have significant consequences on the equilibrium dynamics of the model. First, the long-run growth rate ceases to depend on the markup when it is disentangled from the returns to specialization. Second, the transitional dynamics of the economy is represented by a twodimensional stable manifold, and a simple sufficient condition for stability is provided — though the 

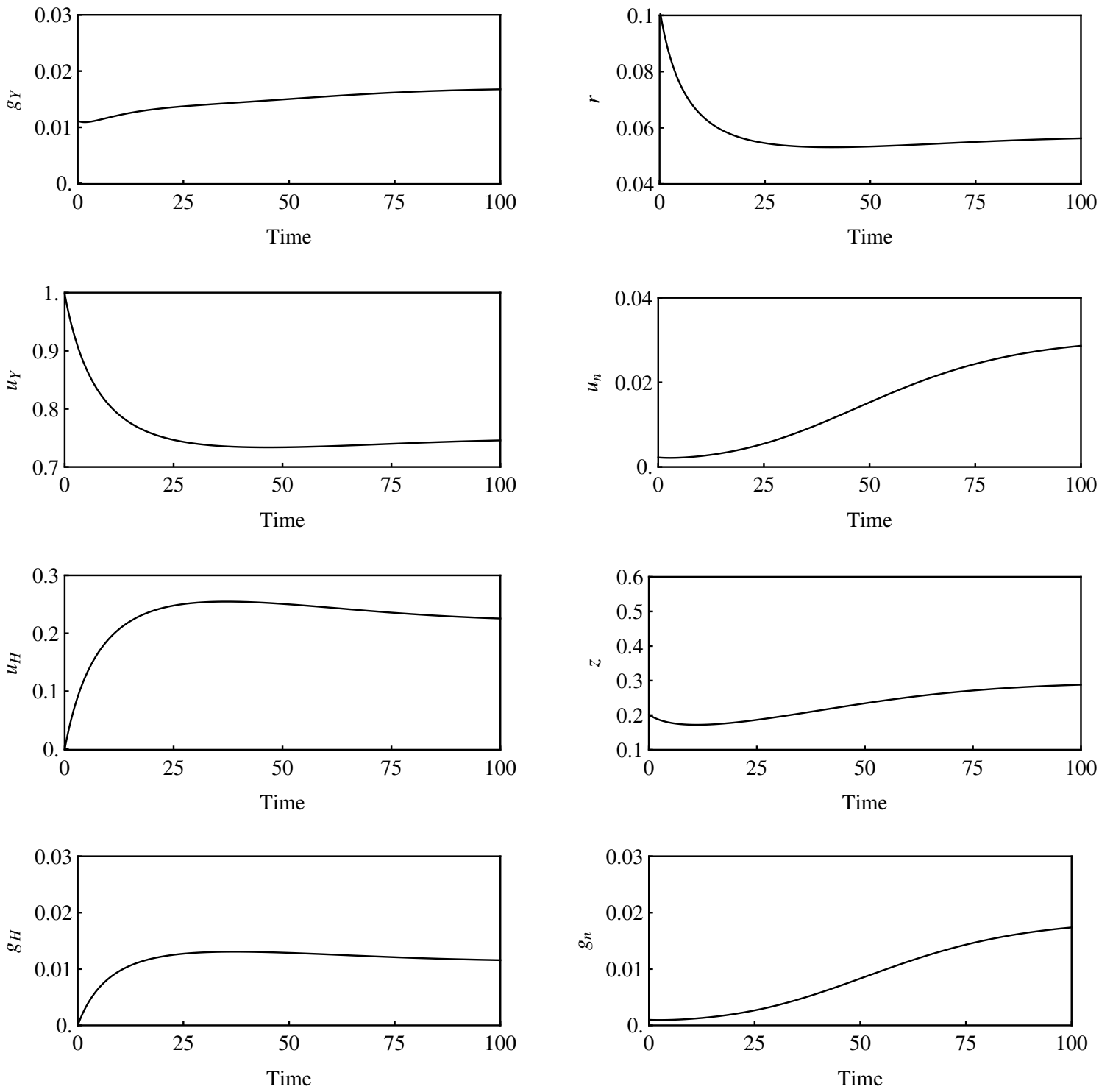

Figure 3: Time paths of representative variables in the model with duplication externalities: innovation-education transition.

Note: Parameter values are shown in Case 2 of Table 3.

instability outcome cannot be ruled out. When both parameters are separated, the markup does not enter in the stability conditions of the general model, whereas it played a prominent role in the corresponding conditions for the standard model. Third, a detailed numerical analysis shows that the steady-state equilibria of the model that disentangles parameters are more prone to be stable than those of the standard model. Furthermore, the introduction of external effects in the $\mathrm{R} \& \mathrm{D}$ sector causes a huge drop in the instability outcomes of both models for the relevant set of parameters.

Simulation results show that the model with spillovers in $R \& D$ is able to replicate a sequence of development in which education precedes innovation as well as a transition in which innovation precedes knowledge formation. Therefore, this model has the potential to generate different development experiences. The additional incorporation of duplication externalities allows the model to 

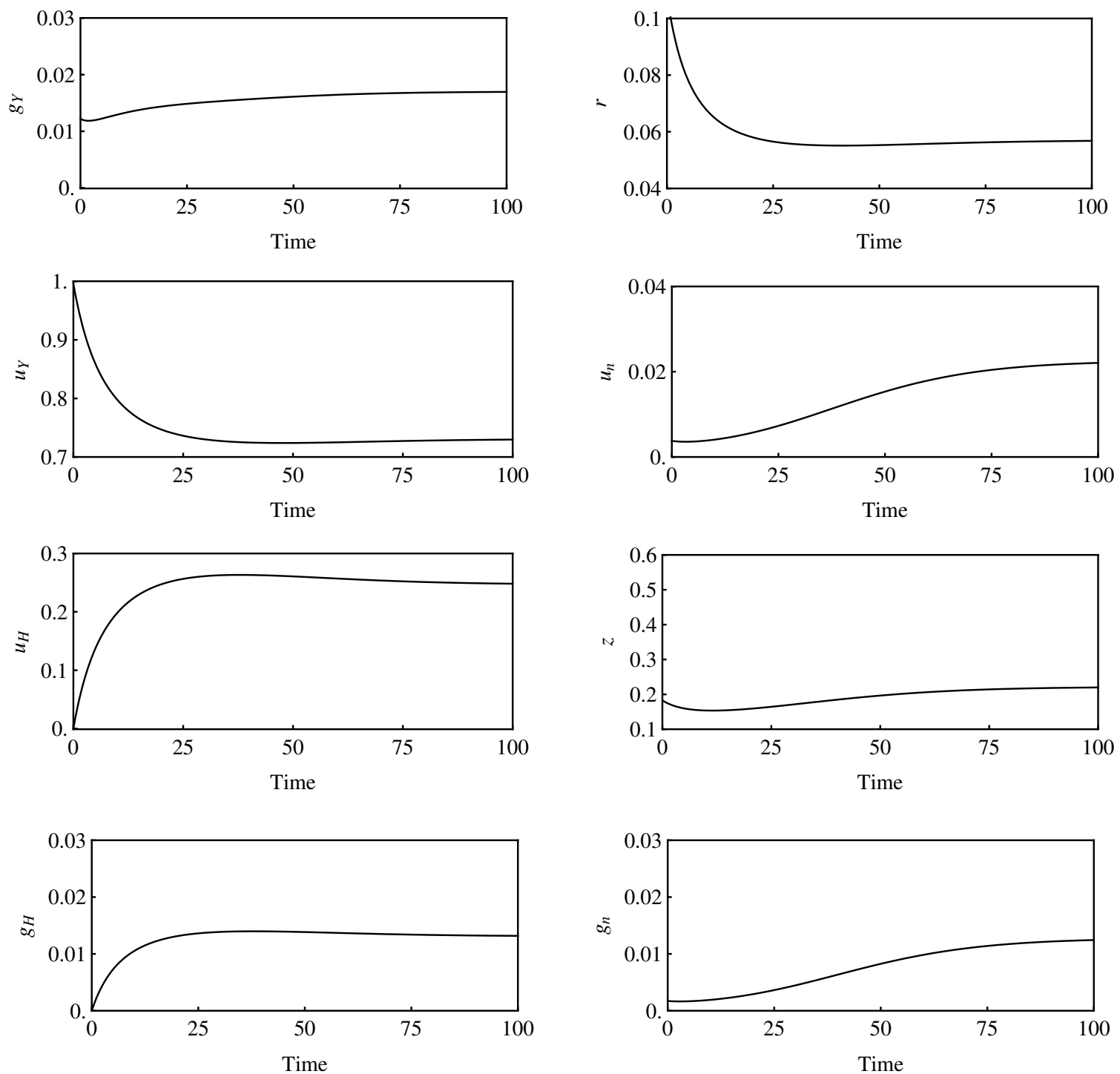

Figure 4: Time paths of representative variables in the model with duplication externalities: innovation-education transition.

Note: Parameter values are shown in Case 3 of Table 3.

replicate important stylized facts of the last century: a roughly constant growth rate of output, an impressive increase of the resources allocated to education, and a (more modest) increase in the resources allocated to R\&D (when compared to human capital). Furthermore, the model can also replicate a decreasing trend in the ratio between outputs and inputs to $R \& D$ while the value of patents increases more than wages. 


\section{Appendix}

\section{A Proofs}

Proof of Proposition 1. Constancy of $\hat{g}_{C}$ implies, by (9), constancy of $r$, i.e., $\hat{g}_{r}=0$. Therefore, $\hat{g}_{Y}=\hat{g}_{K}$, from (17), and $\chi$ is also constant in the steady state, $\hat{g}_{\chi}=0$, from (5). Hence, $\hat{g}_{Y}=\hat{g}_{C}=$ $\hat{g}_{K}$. Constancy of $\hat{g}_{n}$ implies, by (4), constancy of $\psi$, i.e., $\hat{g}_{\psi}=0$. Evaluating (6), (25) and (23) at the steady state we obtain (38), (34) and (36), respectively. Log-differentiating (16) with respect to time, using (36), we get (40). Using (36) and (40) to express $\hat{g}_{C}$ as a function of $\hat{g}_{n}$ in (9), and solving the resulting equation and (26) for $\hat{r}$ and $\hat{g}_{n}$, yields (33) and (35). Finally, (37) results from (24), using (38), and (39) is obtained from (4).

For the interior steady state to be feasible, we must have $0<\hat{u}_{Y}, 0<\hat{u}_{n}, \hat{u}_{Y}+\hat{u}_{n}<1, \hat{r}>0$, $\hat{\chi}>0$ and $\hat{\psi}>0$. Eqs. (36) and (35) entail that condition $0<\hat{u}_{Y}+\hat{u}_{n}=\left(\xi-\hat{g}_{H}\right) / \xi<1$ is satisfied if and only if (41) holds. Since (41) entails that $\theta>1 /(1+M)$, Eqs. (33) and (35) entail that $\hat{r}>0$ and $\hat{g}_{n}>0$ if $\xi>\rho$. Furthermore, $\hat{u}_{Y}$ and $\hat{u}_{n}$ are positive because $\xi-\hat{g}_{H}>0$. Hence, Eq. (39) entails that $\hat{\psi}>0$. Finally, the ratio of consumption to capital can be expressed as

$$
\hat{\chi}=\frac{(1+M) \theta \xi-\rho-\alpha \beta(1+M)(\xi-\rho)}{\alpha \beta[(1+M) \theta-1]} .
$$

The denominator is positive and, as condition (41) can be equivalently rewritten as $(1+M) \theta \xi>$ $\xi+M(\xi-\rho)$, the numerator is also positive because it is greater than $(1-\alpha \beta)(1+M)(\xi-\rho)>0$, and so $\hat{\chi}>0$. Hence, the steady state is feasible.

The transversality condition associated with aggregate wealth is equivalent to $-\hat{r}+\hat{g}_{K}<0$ which, using that $\hat{g}_{K}=\hat{g}_{C}$ and (9), can be rewritten as $(\theta-1) \hat{r}+\rho>0$. If $\theta \geq 1$ it is immediately satisfied. If $\theta<1$, using that $\hat{r}>\xi$, we get that $(\theta-1) \hat{r}+\rho>(\theta-1) \xi+\rho>\rho /(1+M)>0$, where the second inequality follows from (41). The transversality condition associated to human capital, $H$, is equivalent to $-\xi+\hat{g}_{H}<0$. Note that the fulfillment of the transversality condition also ensures convergence of the utility integral because, using that $\hat{g}_{C}=(\hat{r}-\rho) / \theta$, it can be equivalently expressed as $(\theta-1) \hat{g}_{C}+\rho>0$. This completes the proof.

Proof of Proposition 2. It will be useful to rewrite the dynamics of the economy in terms of the variables $r, \chi, g_{n}, z \equiv \epsilon^{1 / \lambda} \psi^{1 / \lambda} u_{Y}$, and $u_{Y}$. Using that $g_{z}=g_{u_{Y}}+(1 / \lambda) g_{\psi}$, we get that the dynamics of the economy is driven by the system (25), (26), and

$$
\begin{aligned}
g_{u_{Y}} & =\left(\frac{1-\alpha}{\alpha \beta}\right) r+\frac{\eta}{\beta} g_{n}-\chi-\xi\left(1-u_{Y}-\frac{g_{n}^{1 / \lambda}}{z} u_{Y}\right)+\frac{\xi}{\beta}, \\
g_{z} & =\left(\frac{1-\alpha}{\alpha \beta}\right) r+\left(\frac{\eta}{\beta}-\frac{1-\phi}{\lambda}\right) g_{n}-\chi+\frac{\xi}{\beta}, \\
g_{g_{n}} & =-\frac{\lambda(1-\alpha) \beta}{(1-\beta)(1-\lambda)} z g_{n}^{1-1 / \lambda}+\frac{\lambda \xi}{1-\lambda}-g_{n}+\frac{\phi}{1-\lambda} g_{n} .
\end{aligned}
$$

Note that the knowledge-ideas ratio, $\psi=H^{\lambda} n^{\phi-1}$, is a predetermined variable. Hence, once the jump variable $u_{Y}$ places on its saddle-path stable trajectory, we have that

$$
z(0)=\epsilon^{1 / \lambda} \psi(0)^{1 / \lambda} u_{Y}(0)
$$

and

$$
r(0)=\alpha \beta n(0)^{\eta}\left[u_{Y}(0) H(0) / K(0)\right]^{1-\beta}
$$


are uniquely determined by the initial values of the predetermined variables $K, H$ and $n$. Hence, the system (25), (26), (A.1), (A.2) and (A.3) features three jump-like variables $-\chi, g_{n}$ and $u_{Y}-$ and two predetermined-like variables $-z$ and $r-$, so that saddle-path stability requires two stable roots.

Linearizing the system (25), (26), (A.1), (A.2) and (A.3) around its steady state $\left(\hat{r}, \hat{\chi}, \hat{g}_{n}, \hat{z}, \hat{u}_{Y}\right)$ gives the following fifth-order system:

$$
\left(\begin{array}{c}
\dot{r} \\
\dot{\chi} \\
\dot{g}_{n} \\
\dot{z} \\
\dot{u}_{Y}
\end{array}\right)=\left(\begin{array}{ccccc}
J_{11} & 0 & J_{13} & 0 & 0 \\
J_{21} & J_{22} & 0 & 0 & 0 \\
0 & 0 & J_{33} & J_{34} & 0 \\
J_{41} & J_{42} & J_{43} & 0 & 0 \\
\cdot & \cdot & \cdot & \cdot & J_{55}
\end{array}\right)\left(\begin{array}{c}
r-\hat{r} \\
\chi-\hat{\chi} \\
g_{n}-\hat{g}_{n} \\
z-\hat{z} \\
u_{Y}-\hat{u}_{Y}
\end{array}\right)=J \cdot\left(\begin{array}{c}
r-\hat{r} \\
\chi-\hat{\chi} \\
g_{n}-\hat{g}_{n} \\
z-\hat{z} \\
u_{Y}-\hat{u}_{Y}
\end{array}\right),
$$

where dots replace those elements that are irrelevant for the analysis, and

$$
\begin{aligned}
J_{11} & =-\frac{1-\beta}{\beta} \hat{r}, & J_{13} & =\frac{\eta}{\beta} \hat{r}, \\
J_{21} & =\left(\frac{1}{\theta}-\frac{1}{\alpha \beta}\right) \hat{\chi} & J_{22} & =\hat{\chi}, \\
J_{33} & =-\left[1-\frac{\phi}{1-\lambda}-\frac{(1-\alpha) \beta \hat{g}_{n}^{-1 / \lambda} \hat{z}}{1-\beta}\right] \hat{g}_{n}, & J_{34} & =-\frac{\lambda(1-\alpha) \beta \hat{g}_{n}^{2-1 / \lambda}}{(1-\beta)(1-\lambda)}, \\
J_{41} & =\frac{1-\alpha}{\alpha \beta} \hat{z}, & J_{42} & =-\hat{z}, \\
J_{43} & =\left(\frac{\eta}{\beta}-\frac{1-\phi}{\lambda}\right) \hat{z}, & J_{55} & =\xi\left(1+\frac{\hat{g}_{n}^{1 / \lambda}}{\hat{z}}\right) \hat{u}_{Y} .
\end{aligned}
$$

The eigenvalues of $J$ are the four eigenvalues of its upper left $4 \times 4$ submatrix (say, $\bar{J}$ ) and its last diagonal element, $J_{55}>0$. Therefore, the number of stable roots of $J$ is equal to that of $\bar{J}$. Using the Routh-Hurwitz theorem, the number of roots of the characteristic equation of $\bar{J}$,

$$
p(x)=x^{4}-\Delta_{3} x^{3}+\Delta_{2} x^{2}-\Delta_{1} x+\Delta_{0}=0,
$$

with negative real parts is equal to the number of variations of sign in the scheme:

$$
\begin{array}{lllll}
1 & \Delta_{3} & \Psi & \Pi \quad \Delta_{0}
\end{array}
$$

where

$$
\begin{aligned}
\Delta_{0} & =\operatorname{det}(\bar{J})=\frac{(1-\alpha) \lambda(\xi-\rho)}{\theta(1-\lambda)} \hat{z} \hat{r} \hat{\chi} \hat{g}_{n}^{1-1 / \lambda}>0, \\
\Delta_{1} & =J_{11} J_{22} J_{33}+J_{13} J_{41} J_{34}-\left(J_{11}+J_{22}\right) J_{34} J_{43}, \\
\Delta_{2} & =\left(J_{11}+J_{22}\right) J_{33}+J_{11} J_{22}-J_{34} J_{43}, \\
\Delta_{3} & =\operatorname{tr}(\bar{J})=J_{11}+J_{22}+J_{33}, \\
\Psi & =\Delta_{2}-\Delta_{1} / \operatorname{tr}(\bar{J}) \\
\Pi & =\Delta_{1}-\left[\Delta_{3} \operatorname{det}(\bar{J}) / \Psi\right] .
\end{aligned}
$$

It is easy to see that

$$
\begin{aligned}
J_{11}+J_{22} & =\left(\hat{r}-\hat{g}_{K}\right)+\left(\frac{1-\alpha}{\alpha \beta}\right) \hat{r}>0, \\
J_{33} & =\left(\xi-\hat{g}_{H}\right)+\frac{\phi}{1-\lambda} \hat{g}_{n}>0,
\end{aligned}
$$


where it has been used that $\hat{r}-\hat{g}_{K}=\xi-\hat{g}_{H}>0$, which can be easily derived from (33), (36) and (40). This means that $\Delta_{3}=\operatorname{tr}(\bar{J})>0$. Given the positivity of the determinant and the trace, there can be at most two variations of sign in the scheme (A.4). Hence matrix $\bar{J}$ may have zero or two roots with negative real parts. This, together with Example 1 in the main text, proves part a).

To prove part b), we first show that a sufficient condition to rule out the case of none stable roots is that $\Delta_{1}<0$. If $\Psi<0$, there are two variations in sign in (A.4) -irrespective of the sign of $\Pi$. If $\Psi>0$ then $\Pi<0$ and, therefore, there are two variations in sign in (A.4). If $\Psi=0$, we substitute it by $\Psi=\iota>0$, and so, $\Pi=\Delta_{1}-\operatorname{tr}(\bar{J}) \operatorname{det}(\bar{J}) / \iota$. Taking the limit as $\iota \rightarrow 0$, we have that $\Pi \rightarrow-\infty$ and, therefore, there are two variations in sign in the scheme (A.4). Given that $J_{11}+J_{22}>0, J_{33}>0$ and $J_{13} J_{41} J_{34}<0$, the expression for $\Delta_{1}$ indicates that a sufficient condition for $\Delta_{1}<0$ is that $J_{43} \leq 0$; i.e., that the condition in the proposition holds.

Proof of Proposition 3. In this case, the dynamics of the economy is driven by the system (25), (26), (A.1) and (A.2), where $g_{n}$ should be substituted with (30). As in the proof of Proposition 2 , it can be easily shown that this dynamic system features two jump-like variables $-\chi$ and $u_{Y}-$ and two predetermined-like variables $-z$ and $r-$, so that saddle-path stability requires two stable roots. Linearizing the dynamics of the economy, we get

$$
\left(\begin{array}{c}
\dot{r} \\
\dot{\chi} \\
\dot{z} \\
\dot{u}_{Y}
\end{array}\right)=\left(\begin{array}{cccc}
J_{11} & 0 & J_{13} & 0 \\
J_{21} & J_{22} & 0 & 0 \\
J_{31} & J_{32} & J_{33} & 0 \\
\cdot & \cdot & \cdot & J_{44}
\end{array}\right)\left(\begin{array}{c}
r-\hat{r} \\
\chi-\hat{\chi} \\
z-\hat{z} \\
u_{Y}-\hat{u}_{Y}
\end{array}\right)=J \cdot\left(\begin{array}{c}
r-\hat{r} \\
\chi-\hat{\chi} \\
z-\hat{z} \\
u_{Y}-\hat{u}_{Y}
\end{array}\right),
$$

where a dot represents an element that is irrelevant for the analysis, and

$$
\begin{array}{ll}
J_{11}=-\frac{1-\beta}{\beta} \hat{r}<0, & J_{13}=\frac{\eta(1-\alpha)}{(1-\beta) \phi} \hat{r}>0, \\
J_{21}=\left(\frac{1}{\theta}-\frac{1}{\alpha \beta}\right) \hat{\chi}, & J_{22}=\hat{\chi}>0, \\
J_{31}=\frac{(1-\alpha)}{\alpha \beta} \hat{z}>0, & J_{32}=-\hat{z}<0, \\
J_{33}=\frac{(1-\alpha)[\eta-\beta(1-\phi)]}{(1-\beta) \phi} \hat{z}, & J_{44}=\hat{r}-\hat{g}_{K}>0 .
\end{array}
$$

Given the structure of $J$, the fourth diagonal element, $J_{44}>0$, is an unstable root. The other three roots are those of its upper left submatrix $3 \times 3$, say $\bar{J}$. Therefore, the number of stable roots of $J$ is equal to that of $\bar{J}$. Using the Routh-Hurwitz theorem, the number of roots of the characteristic equation of $\bar{J}, p(x)=-x^{3}+\Delta_{2} x^{2}-\Delta_{1} x+\Delta_{0}=0$, with negative real parts is equal to the number of variations of sign in the scheme

$$
\begin{array}{llll}
1 & \Delta_{2} & \Psi & \Delta_{0}
\end{array}
$$

where

$$
\begin{aligned}
\Delta_{0} & =\operatorname{det}(\bar{J})=\frac{(1-\alpha)(\xi-\rho)}{\theta \phi \hat{g}_{n}} \hat{r} \hat{\chi} \hat{z}>0, \\
\Delta_{1} & =J_{11} J_{22}-J_{13} J_{31}+\left(J_{11}+J_{22}\right) J_{33}, \\
\Delta_{2} & =\operatorname{tr}(\bar{J})=J_{11}+J_{22}+J_{33} \\
\Psi & =\Delta_{1}-\operatorname{det}(\bar{J}) / \operatorname{tr}(\bar{J})
\end{aligned}
$$


The determinant of $\bar{J}$ is positive, $\Delta_{0}=\operatorname{det}(\bar{J})>0$ and, therefore, there are 0 or 2 stable roots. This, together with Example 2 in the main text, proves part a).

To prove part $\mathrm{b}$ ), we show that a sufficient condition to rule out the case of none stable roots is that $\Delta_{1}<0$. If $\operatorname{tr}(\bar{J})<0$ then there are two variations of sign in the scheme (A.5) -irrespective of the sign of $\Psi$. If $\operatorname{tr}(\bar{J})>0$, then $\Psi<0$ so that there are two variations in sign in (A.5). If $\operatorname{tr}(\bar{J})=0$, we substitute it by $\operatorname{tr}(\bar{J})=\iota>0$, and so, $\Psi=\Delta_{1}-\operatorname{det}(\bar{J}) / \iota$. Taking the limit as $\iota \rightarrow 0$, we have that $\Psi \rightarrow-\infty$ and, therefore, there are two variations in sign in (A.5). Since $J_{11}+J_{22}>0$ (see the proof of Proposition 2), and $J_{13} J_{31}>0$, the expression of $\Delta_{1}$ entails that a sufficient condition for $\Delta_{1}<0$ is that $J_{33} \leq 0$; i.e., that the condition in the proposition holds.

Proof of Proposition 4. In this case, the dynamics of the economy is described by (25), (26) and (28), where $u_{Y}$ and $g_{n}$ should be replaced with (31) and (32), respectively. As in the proof of Proposition 2, it can be easily shown that this dynamic system features one jump-like variable $-\chi$ - and two predetermined-like variables $-\psi$ and $r$-, so that saddle-path stability requires two stable roots. Linearizing the system (25), (26) and (28) around its steady state $(\hat{r}, \hat{\chi}, \hat{\psi})$ yields

$$
\left(\begin{array}{c}
\dot{r} \\
\dot{\chi} \\
\dot{\psi}
\end{array}\right)=\left(\begin{array}{ccc}
J_{11} & J_{12} & 0 \\
J_{21} & J_{22} & 0 \\
\cdot & \cdot & J_{33}
\end{array}\right)\left(\begin{array}{c}
r-\hat{r} \\
\chi-\hat{\chi} \\
\psi-\hat{\psi}
\end{array}\right)=J \cdot\left(\begin{array}{c}
r-\hat{r} \\
\chi-\hat{\chi} \\
\psi-\hat{\psi}
\end{array}\right),
$$

where dots replace those elements that are irrelevant for the analysis, and

$$
\begin{array}{rlrl}
J_{11} & =-\frac{\eta-\alpha \beta(1-\beta+\eta)}{\alpha \beta(\beta-\eta)} \hat{r}, & J_{13}=-\frac{\eta}{\beta-\eta} \hat{r}, \\
J_{21}=\left(\frac{1}{\theta}-\frac{1}{\alpha \beta}\right) \hat{\chi}, & J_{22}=\hat{\chi}, \\
J_{33}=\frac{\xi}{\epsilon \hat{\psi}}\left[\frac{\xi(1-\beta)}{(1-\alpha) \beta}+\hat{g}_{n}\right]>0 . &
\end{array}
$$

Let $\bar{J}$ denote the upper left $2 \times 2$ submatrix of $J$. The structure of $J$ entails that its eigenvalues are its last diagonal element $J_{33}$, which is positive, and the two eigenvalues of $\bar{J}$. The real parts of the eigenvalues of $\bar{J}$ are negative if and only if its determinant is positive and its trace is negative.

The determinant of $\bar{J}$ is

$$
\operatorname{det}(\bar{J})=\frac{\theta(1-\beta+\eta)-\eta}{\theta(\eta-\beta)} \hat{r} \hat{\chi}
$$

Since the numerator of the former expression is positive if condition $(41)$ is satisfied, $\operatorname{det}(\bar{J})>0$ if and only if $\eta>\beta$.

The trace of $\bar{J}$ is

$$
\operatorname{tr}(\bar{J})=\frac{(1-\beta+\eta)\{\theta \xi[\alpha(1-\beta+\eta)-1]-\alpha(\eta-\beta) \xi-\alpha \beta \rho\}+\eta \rho}{\alpha(\eta-\beta)[\theta(1-\beta+\eta)-\eta]} .
$$

The denominator of the trace is positive if condition (41) is satisfied. Hence, there are two cases:

i) If the coefficient of $\theta$ in the numerator of the trace is non-positive; i.e., $\alpha(1-\beta+\eta) \leq 1$, the trace is decreasing in $\theta$. Now, the fact that $\theta>\theta_{\min }$ entails that the trace is negative because

$$
\operatorname{tr}(\bar{J})<\operatorname{tr}(\bar{J})_{\theta=\theta_{\min }}=-\frac{\xi(1-\alpha)(1-\beta+\eta)}{\alpha(1-\beta)(\eta-\beta)}<0 .
$$


ii) If the coefficient of $\theta$ in the numerator of the trace is positive; i.e., $\alpha(1-\beta+\eta)>1$, the numerator of the trace is increasing in $\theta$. Equalizing the numerator of the trace to zero, the value of $\theta$ such that the trace is zero, $\theta_{0}$, is found to be given by (42). Hence, the trace is negative if and only if $\theta<\theta_{0}$. Note that $\theta_{\min }<\theta_{0}$ because

$$
\theta_{\text {min }}-\theta_{0}=\frac{(1-\alpha)(\xi-\rho)}{\xi[1-\alpha(1-\beta+\eta)]}<0 .
$$

Under the former conditions, there are a single unstable and two stable roots. This completes the proof.

\section{References}

Alvarez-Pelaez, M. J. and Groth, C. (2005). Too little or too much R\&D? European Economic Review, 49(2):437-456.

Arnold, L. G. (2000a). Endogenous growth with physical capital, human capital and product variety: A comment. European Economic Review, 44(8):1599-1605.

Arnold, L. G. (2000b). Endogenous technological change: A note on stability. Economic Theory, 16(1):219-226.

Arnold, L. G. (2000c). Stability of the market equilibrium in romer's model of endogenous technological change: A complete characterization. Journal of Macroeconomics, 22(1):69 - 84 .

Arnold, L. G. (2006). The dynamics of the Jones R\&D growth model. Review of Economic Dynamics, 9(1):143-152.

Arnold, L. G. and Kornprobst, W. (2008). Comparative statics and dynamics of the Romer R\&D growth model with quality upgrading. Macroeconomic Dynamics, 12(5):702-716.

Atolia, M. and Buffie, E. F. (2009). Reverse shooting made easy: Automating the search for the global nonlinear saddle path. Computational Economics, 34(3):273-308.

Barro, R. J. and Sala-i-Martin, X. (1995). Economic Growth. McGraw-Hill.

Basu, S. (1996). Procyclical productivity: Increasing returns or cyclical utilization? Quarterly Journal of Economics, 111(3):709-751.

Bénassy, J.-P. (1996a). Monopolistic competition, increasing returns to specialization and output persistence. Economics Letters, 52(2):187-191.

Bénassy, J.-P. (1996b). Taste for variety and optimum production patterns in monopolistic competition. Economics Letters, 52(1):41-47.

Bénassy, J.-P. (1998). Is there always too little research in endogenous growth with expanding product variety? European Economic Review, 42(1):61-69.

Benhabib, J., Meng, Q., and Nishimura, K. (2000). Indeterminacy under constant returns to scale in multisector economies. Econometrica, 68(6):1541-1548.

Benhabib, J. and Perli, R. (1994). Uniqueness and indeterminacy: On the dynamics of endogenous growth. Journal of Economic Theory, 63(1):113-142. 
Brunner, M. and Strulik, H. (2002). Solution of perfect foresight saddlepoint problems: A simple method and applications. Journal of Economic Dynamics and Control, 26(5):737-753.

Coto-Martínez, J., Garriga, C., and Sánchez-Losada, F. (2007). Optimal taxation with imperfect competition and aggregate returns to specialization. Journal of the European Economic Association, 5(6):1269-1299.

de Groot, H. L. F. and Nahuis, R. (1998). Taste for diversity and the optimality of economic growth. Economics Letters, 58(3):291-295.

de Groot, H. L. F. and Nahuis, R. (2002). Optimal product variety and economic growth: The trade-off between internal and external economies of scale. Journal of Economics, 76:1-32.

Eicher, T. S. and Turnovsky, S. J. (1999). Convergence in a two-sector nonscale growth model. Journal of Economic Growth, 4(4):413-428.

Eicher, T. S. and Turnovsky, S. J. (2001). Transitional dynamics in a two-sector non-scale growth model. Journal of Economic Dynamics and Control, 25(1-2):85-113.

Ethier, W. J. (1982). National and international returns to scale in the modern theory of international trade. American Economic Review, 72(3):389-405.

Funke, M. and Strulik, H. (2000). On endogenous growth with physical capital, human capital and product variety. European Economic Review, 44(3):491-515.

Galor, O. (2005). From stagnation to growth: Unified growth theory. In Aghion, P. and Durlauf, S., editors, Handbook of Economic Growth, volume 1, chapter 4, pages 171-293. Elsevier, Amsterdam.

Gómez, M. A. (2005). Transitional dynamics in an endogenous growth model with physical capital, human capital and R\&D. Studies in Nonlinear Dynamics 85 Econometrics, 9(1):Article 5.

Gómez, M. A. (2011a). Duplication externalities in an endogenous growth model with physical capital, human capital, and R\&D. Economic Modelling, 28(1-2):181-187.

Gómez, M. A. (2011b). Stages of economic development in an innovation-education growth model. Studies in Nonlinear Dynamics \& Econometrics, forthcoming.

Gómez, M. A. and Sequeira, T. N. (2011). A note on R\&D spillovers in an endogenous growth model with physical capital, human capital and varieties. Macroeconomic Dynamics, forthcoming.

Grossman, G. M. and Helpman, E. (1991). Innovation and Growth in the Global Economy. MIT Press, Cambridge, MA.

Iacopetta, M. (2010). Phases of economic development and the transitional dynamics of an innovation-education growth model. European Economic Review, 54(2):317-330.

Iacopetta, M. (2011). Formal education and public knowledge. Journal of Economic Dynamics and Control, 35(5):676-693.

Jones, C. I. (1995a). R\&D-based models of economic growth. Journal of Political Economy, 103(4):759-784. 
Jones, C. I. (1995b). Time series tests of endogenous growth models. Quarterly Journal of Economics, 110(2):495-525.

Jones, C. I. (2002). Sources of U.S. economic growth in a world of ideas. American Economic Review, 92(1):220-239.

Jones, C. I. and Williams, J. C. (2000). Too much of a good thing? The economics of investment in R\&D. Journal of Economic Growth, 5(1):65-85.

Kim, J. (2004). What determines aggregate returns to scale? Journal of Economic Dynamics and Control, 28(8):1577-1594.

Kortum, S. (1993). Equilibrium R\&D and the patent-R\&D ratio: U. S. evidence. American Economic Review, 83(2):450-457.

Maddison, A. (2001). The World Economy: A millenial perspective. OECD Development Centre.

Martínez-García, M. P. (2003). The general instability of balanced paths in endogenous growth models: the role of transversality conditions. Journal of Economic Dynamics and Control, 27(4):599618.

Mino, K. (2001). Indeterminacy and endogenous growth with social constant returns. Journal of Economic Theory, 97(1):203-222.

Norrbin, S. (1993). The relation between price and marginal cost in U.S. industry: A contradiction. Journal of Political Economy, 101(6):1149-1164.

Romer, P. M. (1990). Endogenous technological change. Journal of Political Economy, 98(5):S71S102.

Sequeira, T. N. (2011). R\&D spillovers in an endogenous growth model with physical capital, human capital, and varieties. Macroeconomic Dynamics, 15(2):223-239.

Sequeira, T. N. and Reis, A. B. (2007). Human capital and overinvestment in R\&D. Scandinavian Journal of Economics, 109(3):573-591.

Steger, T. M. (2005). Non-scale models of R\&D-based growth: The market solution. The B.E. Journal of Macroeconomics, 5(1):Article 3.

Stokey, N. L. and Rebelo, S. (1995). Growth effects of flat-rate taxes. Journal of Political Economy, 103(3):519-550. 